\title{
Correlation Between Joint Position Sense, Threshold To Detection Of Passive Motion Of The Knee Joint And Walking Speed Of Post-Stroke Patient
}

\author{
Kurnia Imanawanto ${ }^{1}$, Meisy Andriana ${ }^{1}$, Rwahita Satyawati $^{1}$ \\ email: meisy.andriana@fk.unair.ac.id \\ ${ }^{1}$ Physical Medicine and Rehabilitation, Universitas Airlangga, Dr.Soetomo General Academic Hospital, Surabaya, Indonesia
}

\begin{abstract}
Background: Sensorimotor dysfunction such as muscle weakness, impaired sensation, changes in muscle tone, reduced movement control are common in stroke patients. Locomotion function requires continuous afferent sensory input. Impairment of sensory function can hinder the ability to recruit muscles during walking. Patients generally walk slowly, cadence, step and stride length are shorter and the double phase of support increases.

Goal: Analyzing the correlation between the propioceptive function of the knee joint and walking speed in post-stroke patients, who are treated at the PMR Outpatient Clinic of Dr. Soetomo General Academic Hospital Surabaya.

Methode: An observational analytic cross sectional study, nine subjects measured walking speed (10MWT), joint position sense (JPS) and threshold to detetction of passive motion (TTDPM) of the knee joint, in the period March - November 2020..

Results: There is a moderate correlation between 10MWT and JPS $15^{0}$ on the non-paretic side (r 0.676; $\mathrm{p}=0.045$ ) and JPS $30^{\circ}$ on the paretic side ( $\left.\mathrm{r} 0.668 ; \mathrm{p}=0.049\right)$. And there is a strong correlation between 10MWT and JPS $60^{\circ}$ on the paretic side ( $\mathrm{r} 0.824 ; \mathrm{p}=0.006$ ). However, there is no significant correlation between 10MWT and TTDPM.

Conclusion: There is correlation between the propioceptive function of the knee joint and the walking speed of post-stroke patients. The smaller the percentage of joint position error, the stronger the correlation with walking speed.
\end{abstract}

Keywords: 10 meter walking test; joint position sense; threshold to detection of passive motion; knee joint; proprioception; stroke

\section{Introduction}

Stroke is a neurological deficit cause sensorimotor dysfunction such as muscle weakness, impaired sensation, changes in muscle tone, reduced control of movement is common in stroke patients (Zorowitz et al, 2015; Coupland et al, 2017; Sader et al, 2018). The frequency of sensory deficits up to $50 \%$ occurs in the acute phase of stroke, and up to $25 \%$ occurs in the chronic phase (Harvey dan Elliot, 2008). Impaired motor control is a frequent manifestation and plays a role in decreasing the ability to walk. The first six weeks, $40 \%$ survivor have severe motor weakness that limits walking (Sullivan, 2009; Sader et al., 2018).

The human walking normal pattern shows repetitive characteristics as gait cycle. The pattern of acceleration and deceleration is precisely regulated by the anti-gravity muscles (Pease dan Bowyer, 2010; Joseph dan Benjamin, 2019). If its abnormal is depends on the area of brain damage and the affected systems such as the motor, sensory, balance, perceptual coordination and visual systems (Bassile et al., 2016). They 
walk slowly, cadence, step and stride length are shorter and the double support phase is increased (Hausdorff dan Alexander, 2005; Sader et al., 2018).

The goals of stroke rehabilitation are to achieve a maximal level of independent activity, facilitate neurological recovery, minimize disability, and reintegrate back into society. These goals can be achieved through exercises and therapies (Stein dan Brandstater, 2010; Lloyd A et al., 2018). Walking requires voluntary motor function, especially hip extensor muscle group. These stabilize the hip in an extended position, and stabilize the knee extended by pulling the hip to back (Anderson, 1990; Dubey et al., 2018). The knee muscles is need to be trained, act as are the flexor and extensor muscles of the knee joint (Flansbjer, 2006).

In the terminal swing phase, stroke patient have inability to extend the knee so that foot is in contact with the floor when the knee is flexed, there will be a decrease in the step length of the paretic side (Bassile et al., 2016). Normal person's EMG recording on knee muscle activity shows a percentage of $45 \%$ in the knee extensor muscle group and 35\% in the knee flexor muscle group (Pease dan Bowyer, 2010). The knee muscle contraction has eccentric contractions and concentric contractions in certain gait phases (Kerrigan dan Edelstein, 2001). There is not clear what contributes to the decreased ability to walk after a stroke (Flansbjer, 2006; Fujita et al., 2018).

Impairment of sensory function can impede the recruitment ability of the muscles during walking. Patients tend to be slower in achieving functional ability and fail to achieve optimal recovery. Research evidence shows that tactile sensation and joint position sense (JPS) have different roles in controlling locomotion. There are studies that assess changes in proprioceptive function in normal people showing changes in gait patterns. Animal studies have shown that impaired knee position sensation is associated with muscle recruitment during walking (Lin, 2005). This study was conducted to measure the correlation between knee joint proprioceptive function and walking speed of post-stroke patients, may it be used as the basis for appropriate interventions to therapy of post-stroke patients.

\section{Methode and Materials}

\subsection{Subject}

The subjects of subacute and chronic stroke were 9 people in the PMR Outpatient Clinic of Dr. Soetomo General Academic Hospital Surabaya between March 2020 to November 2020. The inclusion criteria are as follows: (1). Age 30-60 years, (2). Sub acute and chronic stroke phases, (3). Initial attack of stroke or repetition, (4). Able to walk with/or without a walker at least 10 meters, (5). Able to follow and understand simple verbal instructions, (6). Functional hearing and vision, (7). Modified Asworth Scale spasticity maximum score of 2 on knee flexor and extensor muscles, (8). Willing to sign an informed consent. The walking speed is measured using 10 meter Walk Test (10m-WT), consist of comfortable walking speed (CWS) and fast walking speed (FWS) with results in meters/second.

\subsection{Joint Position Sense (JPS)}

The subject was in a sitting position with the knee free on flexion and extension, a strap was attached to the distal tibia connected to the dynamometer arm. The patient will feel the knee joint position at $15^{\circ}, 30^{\circ}$ and $60^{\circ}$ flexion angles, then perform five leg repositions at that angle with eyes closed. The difference between the target angle and the joint repositioning angle (absolute error/AE) is calculated in degrees (absolute error normal value: $2.5^{\circ}$ ). 
2.3 Threshold to detection of passive motion (TTDPM)

The subject was in a sitting position with the knee free of flexion and extension, a strap was attached to the distal tibia connected to the dynamometer arm. Subjects were asked to press the stop button when they felt the knee joint movement generated by the machine at a speed of 10 per second. The TTDPM value is the duration of the subject can feel the movement of the knee joint in seconds. It was repeated 5 times, the average value was taken. Examination was performed on both paretic and non-paretic (normal value for TTDPM: 2.5-4 seconds).

\section{Results}

Table 1. Subject Characteristics

\begin{tabular}{|c|c|c|c|c|c|}
\hline Characteristics & n & Min & Max & Mean \pm SD & $\mathbf{p}$ \\
\hline Age (year) & 9 & 30 & 57 & $46,3 \pm 10,3$ & 0,091 \\
\hline \multicolumn{6}{|l|}{ Gender } \\
\hline Male & $5(55,6 \%)$ & - & - & - & \\
\hline Female & $4(44,4 \%)$ & - & - & - & \\
\hline Infarct stroke & $7(77,8 \%)$ & - & - & - & \\
\hline Haemorrhagic stroke & $2(22,2 \%)$ & - & - & - & \\
\hline Onset Stroke (months) & 9 & 2,5 & 48 & $15,2 \pm 19$ & 0,001 \\
\hline$>6$ month & $5(55,6 \%)$ & 2,5 & 4 & $3,1 \pm 0,7$ & 0,224 \\
\hline$<6$ month & $4(44,4 \%)$ & 10 & 48 & $22,7 \pm 20,8$ & 0,063 \\
\hline Right Hemiparese & $3(33,3 \%)$ & & & & \\
\hline Left Hemiparese & $6(66,6 \%)$ & & & & \\
\hline BMI $\left(\mathrm{kg} / \mathrm{m}^{2}\right)$ & & 20,2 & 31,3 & $24,5 \pm 3,68$ & 0,308 \\
\hline MMT knee flexor & & 3 & 4 & $3,55 \pm 0,52$ & 0,000 \\
\hline MMT knee extensor & & 3 & 4 & $3,67 \pm 0,5$ & 0,000 \\
\hline
\end{tabular}

Description: significance of $\mathrm{p}<0.05$ using the normality test with Saphiro-Wilk

Table 2. Absolute Error of Joint Position Sense

\begin{tabular}{|c|c|c|c|c|c|c|}
\hline & $\begin{array}{l}\text { AE JPS } 15^{\circ} \\
(\text { Mean } \pm S D)\end{array}$ & $\mathrm{p}$ & $\begin{array}{l}\text { AE JPS } 30^{\circ} \\
(\text { Mean } \pm \text { SD) }\end{array}$ & $\mathrm{p}$ & $\begin{array}{l}\text { AE JPS } 60^{\circ} \\
(\text { Mean } \pm S D)\end{array}$ & $\mathrm{p}$ \\
\hline Paretic & $4,06 \pm 4,79$ & 0,029 & $6,82+3,71$ & 0,450 & $6,03 \pm 4,22$ & 0,677 \\
\hline Non-Paretic & $6,33+4,93$ & 0,046 & $6,68+3,86$ & 0,005 & $6,84+5,75$ & 0,389 \\
\hline
\end{tabular}

Description: significance of $\mathrm{p}<0.05$, normality test with Saphiro-Wilk

Table 3 Absolute Error of TTDPM

\begin{tabular}{lcc}
\hline & $\begin{array}{c}\text { AE TTDPM (seconds) } \\
(\text { Mean } \pm \text { SD) }\end{array}$ & $p$ \\
\hline Paretic & $8,92 \pm 6,46$ & 0,155 \\
Non-Paretic & $5,96 \pm 3,72$ & 0,693 \\
\hline
\end{tabular}

Description: significance of $\mathrm{p}<0.05$, normality test with Saphiro-Wilk 
Table 4 Comparation of TTDPM based on stroke onset

\begin{tabular}{ccccc}
\hline \multirow{2}{*}{ Stroke onset } & \multicolumn{4}{c}{ AE TTDPM (seconds) } \\
\cline { 2 - 5 } & paretic & $\mathrm{p}$ & non-paretic & $\mathrm{p}$ \\
\hline$\leq 6$ month & $12,2 \pm 6,5$ & 0,473 & $7,35 \pm 4,3$ & 0,625 \\
\hline$>6$ month & $4,72 \pm 3,4$ & 0,013 & $4,22 \pm 2,1$ & 0,503 \\
\hline Description: significance of $\mathrm{p}<0.05$, normality test with Saphiro-Wilk
\end{tabular}

Table 5 shows the measurement of walking speed using the 10 meter walk test method, measuring comfortable walking speed (CWS) and fast walking speed (FWS). Correlation test used walking speed data with an average value of $0.69 \pm 0.27$ meter/second.

Table 5 Walking speed of stroke

\begin{tabular}{lcccccc}
\hline & $\begin{array}{c}\text { CWS } \\
(\text { Mean } \pm \text { SD })\end{array}$ & p & $\begin{array}{c}\text { FWS } \\
(\text { Mean } \pm \text { SD) }\end{array}$ & p & $\begin{array}{c}\text { Walking speed } \\
(\text { Mean } \pm \text { SD) }\end{array}$ & $p$ \\
\hline $\begin{array}{l}\text { Speed } \\
\text { (meter/seconds) }\end{array}$ & $0,57 \pm 0,25$ & 0,157 & $0,81 \pm 0,32$ & 0,173 & $0,69 \pm 0,27$ & 0,117 \\
\hline
\end{tabular}

Description: significance of $\mathrm{p}<0.05$ using the normality test with Saphiro-Wilk

Table 5 shows a moderate and strong correlation between walking speed and JPS at an angle of $15^{0}$ on the non-paretic side of the leg and JPS at an angle of $30^{\circ}$ and $60^{\circ}$ on the paretic side with significant values $(\mathrm{p}<$ $0.05)$. For other variables did not show a significant correlation $(\mathrm{p}>0.05)$..

Table 5 Correlation between JPS and TTDPM to Walking speed

\begin{tabular}{lcc}
\hline \multirow{2}{*}{ Variable } & \multicolumn{2}{c}{ average walking speed } \\
\cline { 2 - 3 } & $\mathbf{r}$ & $\mathbf{P}$ \\
\hline AE JPS $15^{0}$ paretic & 0,214 & 0,580 \\
AE JPS $15^{\circ}$ non- paretic & 0,676 & $0,045^{*}$ \\
AE JPS $30^{0}$ paretic & 0,668 & $0,049^{*}$ \\
AE JPS $30^{\circ}$ non- paretic & 0,371 & 0,325 \\
AE JPS $60^{\circ}$ paretic & 0,824 & $0,006^{*}$ \\
AE JPS $60^{\circ}$ non- paretic & 0,556 & 0,120 \\
AE TTDPM paretic & $-0,520$ & 0,151 \\
AE TTDPM non- paretic & $-0,289$ & 0,451 \\
\hline
\end{tabular}

$* \mathrm{p}<0.05$ 
Table 6 Correlation between TTDPM to Walking speed

\begin{tabular}{lll}
\hline \multirow{2}{*}{ Variable } & \multicolumn{2}{c}{ average walking speed } \\
\cline { 2 - 3 } & correlation & p \\
\hline AE TTDPM paretic $\leq 6$ months & $-0,700$ & 0,188 \\
AE TTDPM non-paretic $\leq 6$ months & $-0,669$ & 0,217 \\
AE TTDPM paretic $>6$ months & 0,600 & 0,400 \\
AE TTDPM non- paretic $>6$ months & 0,943 & 0,057 \\
\hline
\end{tabular}

The difference of relationship between JPS and TTDPM was analyzed by paired t-test with a significance of $\mathrm{p}<0.05$, a strong correlation was obtained between AE JPS $30^{\circ}$ paretic side and AE TTDPM paretic side with $\mathrm{r}=-0.685(\mathrm{p}=0.042)$.

Table 7 shows a moderate effect size between JPS $15^{\circ}$ paretic side and non-paretic TTDPM, but others variables have small effect sizes.

Table 7 Effect size between JPS and TTDPM

\begin{tabular}{lcc}
\hline & $\begin{array}{c}\text { AE TTDPM } \\
\text { paretic } \\
\text { d }\end{array}$ & $\begin{array}{c}\text { AE TTDPM } \\
\text { non-paretic } \\
\text { d }\end{array}$ \\
\hline AE JPS $15^{0}$ paretic & $-0,533$ & $-0,336$ \\
AE JPS $15^{\circ}$ non-paretic & $-0,217$ & $-0,119$ \\
AE JPS $30^{\circ}$ paretic & $-0,223$ & 0,129 \\
AE JPS $30^{\circ}$ non-paretic & $-0,375$ & $-0,138$ \\
AE JPS $60^{\circ}$ paretic & $-0,310$ & 0,012 \\
AE JPS $60^{\circ}$ non-paretic & $-0,202$ & 0,119 \\
\hline
\end{tabular}

Description: $d=$ effect size using Cohen' d formula

\section{Discussion}

Table 2 shows the absolute error of JPS in the study more than $2.5^{0}$ both at knee flexion angles of $15^{\circ}, 30^{\circ}$ and $60^{\circ}$. Both sides have the same error value of about $6^{\circ}$, except the paretic side when the knee is flexed at an angle of $15^{\circ}$ which has an error value of $4^{0}$. In percentage terms, there are errors up to $26.7 \%$ and $42.2 \%$ at an angle of $15^{\circ}, 22.2 \%$ and $22.7 \%$ at an angle of $30^{\circ}$ and $10 \%$ and $11.4 \%$ at an angle of $60^{\circ}$. Research by Buchanan (2019) explains that compared to healthy people, stroke patients show increased joint position errors and variability during active matching. There is also a proprioceptive deficit on the non-paretic side. This should not happen because the knee joint is controlled by a healthy hemisphere of the brain. Research by Piriyaprasarth et al determined the number of repetitions of the knee joint proprioception assessment, finding that the contralateral limb (non-paretic) also experienced an error of up to 15.6\% (Piriyaprasarth et al., 2009).

Chronic stroke patients experience postural instability which results in asymmetry of body loading towards the non-paretic side (de Kam et al., 2017). Research by Sub Kim et al examined 14 seniors without hemiplegia and 12 seniors with hemiplegia measured by vertical ground reaction force, data obtained that the support moment decreases on the paretic side and increases on the non-paretic side both in the hip, knee and ankle 
joints (Kim and Kim, 2016). Imbalance of loading on joints can increase joint wear and tear. A study by Aderibigbe et al examined the knee joint sonography of 48 stroke patients compared to 26 healthy subjects. The findings of joint soft tissue arthritis, medial and lateral cartilage thickening of the femur, bone border irregularities on both the paretic and non-paretic sides (Aderibigbe et al., 2020). A study by Al-Dadah et al conducted an assessment of proprioceptive function in 50 patients with articular cartilage lesions of the knee joint and found a proprioception deficit compared to the control group and the deficit was also found on the healthy side of the knee (Al-Dadah et al., 2020). In this study, the joint position error was relatively larger than the non-paretic side. This can be explained that the knee joint on the healthy side is overloaded and affects the occurrence of knee osteoarthritis which can result in a greater proprioceptive deficit than the paretic side.

Table 3 shows the TTDPM error value in the study more than 4 seconds, with a greater error value on the paretic side than on the non-paretic side. This shows a sign of a deficit in proprioceptive function in the knee joint (Buchanan, 2019). Research by Neissen et al. (2008) found that in stroke patients there was a deviation in the TTDPM value, with the error on the paretic side being greater than on the non-paretic side.

Subjects showed a lower walking speed than healthy people. Healthy person walking speed had an average value of 1.13 meters/second, while in this study the average walking speed was $0.69 \pm 0.27$ meters/second. Compared to Whittle (2007), this value is also still lower than the walking speed of women aged 65-80 years. Sader et al (2018) conducted a meta-analysis of gait in stroke patients in the subacute phase, it was found that the prevalence of gait disorders was up to $63.6 \%$ with an average walking speed of 0.34 meter/second or a decrease in speed of more than $70 \%$ normal walking speed.

Table 5 shows a moderate correlation between JPS $15^{0}$ non-paretic side $(r=0.676 ; \mathrm{p}=0.045)$ and JPS $30^{\circ}$ paretic side $(\mathrm{r}=0.668 ; \mathrm{p}=0.049)$ with an average walking speed. There is a strong correlation between JPS $60^{\circ}$ paretic side $(\mathrm{r}=0.824 ; \mathrm{p}=0.006)$ and the mean walking speed. Hwang et al. (2010) assessed knee proprioception at flexion of $30^{\circ}$ and $60^{\circ}$ using an isokinetic dynamometer and stated that this assessment is useful for the diagnosis and proprioceptive monitoring of the knee joint in hemiplegic patients due to brain injury. The study compared 30 healthy subjects with 24 hemiplegic subjects, measured the joint position of $30^{\circ}$ and $60^{\circ}$, and detected passive motion using an isokinetic dynamometer. The result is a proprioceptive deficit on the paretic side and on the non-paretic side.

The research findings showed that at $15^{\circ}$ knee flexion, the non-paretic side actually experienced a larger error than the paretic side (42.2\% compared to $26.7 \%$ ), and had a moderate correlation with walking speed. This information can help clinicians to be aware that proprioception deficits in stroke patients can also occur on the healthy side (Hwang et al., 2010). In healthy people the knee joint experiences $15^{0}$ flexion during the loading response and mid-stance phases. This phase plays a role in continuing the acceleration of the body forward and lowering the center of gravity so that it can reduce energy expenditure and the stance phase becomes efficient (Kerrigan and Edelstein, 2001). Patients with hemiplegic gait will experience hyperextension of the knee joint when walking in the mid-stance phase, because in the loading response phase the knee joint on the paretic side remains extended (no knee flexion of $15^{\circ}$ ) even though an ankle rocker occurs (Bassile and Hayes, 2016).

Healthy person's knee joint has $30^{0}-45^{0}$ flexion during the pre-swing phase. In this phase, the knee joint changes very quickly from almost full extension in the heel-off phase to flexion to close to $40^{0}$ during the toeoff phase (Kerrigan and Edelstein, 2001). Whereas in stroke patients, due to hyperextension during the midstance phase, the knee joint becomes less flexed when entering the pre-swing phase, causing the toe clearance condition to be not optimal, so the swing phase is not efficient. Furthermore, the knee joint enters the midswing phase so that the knee is flexed up to $60^{\circ}$ (Carr and Roberta, 2003; Bassile and Hayes, 2016).

The mid-swing phase will take place quickly until the knee is flexed about $60^{\circ}$. This flexion facilitates foot clearance and leg acceleration to immediately enter the terminal swing phase, so that the optimal step length can be achieved. In this study, there was a strong relationship between JPS $60^{\circ}$ and walking speed. From the 
knee joint kinematics when walking, it can be seen that there is a correlation between the angle of the joint position and the walking speed of post-stroke patients. The research shows that the smaller the percentage of error in the joint position, the stronger the correlation with walking speed.

Table 5 also shows no significant correlation value between TTDPM and walking speed on both the paretic $(\mathrm{r}=-0.520 ; \mathrm{p}=0.151)$ and non-paretic $(\mathrm{r}=-0.289 ; \mathrm{p}=0.451)$. Its shows that the larger the TTDPM error, the slower the running speed. In stroke patients, proprioceptive disturbances occur with superficial lesions in the cerebral cortex, while proprioceptive receptors and peripheral afferent pathways are still intact. So in stroke patients the increased time to detect knee joint motion is due to impaired central processing of proprioceptive input. Gait as a reciprocal movement, requires coordination of sensory input and motor output. Slow sensory input (proprioception) can interfere with muscle contractions required for contraction at certain phases of the gait (Bassile and Hayes, 2016).

The insignificant correlation can be caused by several influencing factors, for example because the variable assessed is walking speed, which is a complex movement involving many components of muscles, joints, balance, visual and nervous system. While in this study only focus on one body segment (knee joint) and also only proprioceptive measurements. In addition, research measurement techniques are also used. Research by Piriyaprasarth et al showed that at least 10 measurements of positive function were carried out so as not to increase the percentage of error. In this study, the JPS and TTDPM measurements were carried out with five repetitions (Piriyaprasarth et al., 2009).

Table 6 shows no significant correlation between walking speed and TTDPM based on stroke onset (less than 6 months and more than 6 months). The correlation was negative at the onset of TTDPM less than 6 months, suggesting that the duration of detection of passive knee motion is associated with slower walking speed. If it has been more than 6 months the body quickly recognizes passive knee movements associated with increased walking speed.

Based on table 4, the detection of passive motion of the paretic side of the knee joint takes longer than the non-paretic side at stroke onset of less than 6 months, and both sides still show elongation because it takes more than 4 seconds. The data also shows changes in motion detection time approaching normal values at stroke onset of more than 6 months on both paretic and non-paretic sides, although the TTDPM value is still more than 4 seconds. Semrau et al. conducted a proprioception examination, obtained a deficit of up to $48 \%$ $68 \%$ which was then assessed at 6 weeks, 12 and 26 weeks. Recovery of proprioception which is grouped into early, late and incomplete recovery (Semrau et al., 2015). While the recovery of motor function during the first 6 weeks after stroke, most patients can regain the ability to walk, but around $40 \%$ experience severe motor weakness that limits the ability to walk functionally (Sullivan, 2009; Sader et al., 2018).

The study also found a strong correlation between AE JPS 30 paretic side and AE TTDPM paretic side with $r=-0.685(\mathrm{p}=0.042)$. The negative correlation means that the smaller the joint position error at $30^{\circ}$, the greater the time error required to detect passive knee joint motion. Coincidentally JPS $30^{0}$ paretic side also correlated (moderately) with walking speed (Table 5). Healthy people walking, knee joint flexion of $30^{\circ}$ occurs in the swing phase, at the initial and mid-swing phases, while in stroke patients there is limited flexion (less than $30^{\circ}$ ). As a result, stroke patients cannot perform sufficient clearance and compensate with hip circumduction movements to move forward (Bassile and Hayes, 2016). A study by Gorst et al. (2019) found that $19 \%$ of chronic stroke patients had impaired proprioception in the AGB that affected the ability to mobilize. The study also revealed that two-point discrimination deficits were more dominant (55\%) influencing gait and balance in stroke patients.

The findings in this study are in line with the study by Fujita et al. (2020) which stated that proprioception and knee extension strength were related to gait in stroke patients. The study was conducted retrospectively on 
108 stroke patients and analyzed the relationship between knee extension strength, sensory function and walking ability. This study is different from the study by Li et al. (2015) which also found a non-significant correlation between motion detection and knee joint position detection. The study assessed 30 healthy people, measured using the joint angle reset method, motion minimum threshold measurement and force sense reproduction method on the knees and obtained a correlation coefficient of 0.29.

Based on Table 7, the effect size between JPS and TTDPM is greatest for the variables AE JPS $15^{\circ}$ and AE TTDPM on the paretic side. The research findings show that the largest absolute error also occurs in the TTDPM variable (Table 4). Knee flexion of $15^{0}$ in normal gait occurs during the mid-stance phase, which has benefits for the absorption of force immediately after the heel strike and loading response, and also shortens the body's center of mass, which is beneficial for energy conservation during walking. However, stroke patients experience hyperextension in the mid-stance phase, causing prolonged phase and slower walking speed.

The limitation of this study are proprioception assessment is only carried out on the knee joint, while walking involves the hip and ankle, so measurements should also be taken to determine the relationship with walking speed. The study also did not assess the condition of the knee joint before the examination, which might affect proprioceptive function, for example the condition of knee osteoarthritis.

\section{Conclussion}

There is correlation between knee joint JPS and walking speed of post-stroke patients. However, there is no correlation between knee joint TTDPM and walking speed of post-stroke patients. The results showed that the greater the time needed to detect knee joint motion, the lower the walking speed of post-stroke patients. The study also found a difference between the relationship between JPS and TTDPM of the knee joint with walking speed of post-stroke patients.

\section{References}

Aderibigbe, A.S., Famurewa, O.C., Komolafe, M.A., Omisore, A.D. and Adetiloye, V.A., 2020. Sonographic soft tissue arthritic changes associated with post-stroke hemiplegic knee pain: utility of musculoskeletal ultrasound in a resource-limited setting. Polish journal of radiology, 85 , p.e45.

Al-Dadah, O., Shepstone, L. and Donell, S.T., 2020. Proprioception deficiency in articular cartilage lesions of the knee. Knee Surgery \& Related Research, 32, pp.1-7.

Anderson TP, 1990. Rehabilitation of Patients with Completed Stroke. In Krusen's Handbook of Physical Medicine and Rehabilitation. $4^{\text {th }}$ Ed. Philadelphia: WB Saunders Company

Bassile CC dan SM Hayes, 2016. Gait Awareness In: Stroke Rehabilitation Gillen: a Function-Based Approach. $4^{\text {th }}$ Ed. Missouri: Elsevier. pp194-223

Buchanan, J. 2019. Assessment of Proprioceptive Acuity in Patients with Stroke with Left Hemiplegia Compared to Healthy Controls.

Coupland AP, Ankur T, Mahim IQ, Harri J, Alun HD, 2017. The Definition of Stroke. Journal of the Royal Society of Medicine; Volume: 110 issue:1, page(s): 9-12. https://doi.org/10.1177/2F0141076816680121

de Kam, D., Kamphuis, J.F., Weerdesteyn, V. and Geurts, A.C., 2017. The effect of weight-bearing asymmetry on dynamic postural stability in people with chronic stroke. Gait \& posture, 53, pp.5-10.

Flansbjer UB, Lexell J, dan Brogardh C, 2012. Isokinetic knee extension and flexion strength in individuals with hemiparetic after stroke. Isokinetics and Exercise Science 20,91-97. doi: 10.3233/IES-2012-0446

Fujita K, Hori H, dan Kobayashi Y, 2018. Contribution of muscle activity at different gait phases for improving walking performance in chronic stroke patients with hemiparetic. Journal of Physical Therapy Science; 30(11), 1381-1385. doi:10.1589/jpts.30.1381

Fujita, T., Ohashi, Y., Kurita, M., Yamane, K., Yamamoto, Y., Sone, T., and Iokawa, K. (2020). Functions necessary for gait independence in patients with stroke: A study using decision tree. Journal of Stroke and Cerebrovascular Diseases, 29(8), 104998.

Joseph BW dan Benjamin JD, 2019. Principles of Normal and Pathologic Gait. Atlas of Orthoses and Assistive Devices. 5th Ed. pp 4962.e. https://doi.org/10.1016/B978-0-323-48323-0.00004-4

Gorst, T., Rogers, A., Morrison, S.C., Cramp, M., Paton, J., Freeman, J. and Marsden, J., 2019. The prevalence, distribution, and functional importance of lower limb somatosensory impairments in chronic stroke survivors: a cross sectional observational study. Disability and rehabilitation, 41(20), pp.2443-2450. 
Hausdorff JM, NB Alexander, 2005. Gait Disorders: Evaluation and Management. $1^{\text {st }}$ Ed. UK: CRC Press.

Hwang, J. S., Lee, D. S., Cho, Y. J., Han, N. M., \& Kim, H. D. 2010. Measurement of Proprioception of the Knee in Hemiplegic Patients Using an Isokinetic Dynamometer. Journal of the Korean Academy of Rehabilitation Medicine, 34(1), 27-33.

Kerrigan DC dan Edelstein JE, 2001. Gait. In Downey and Darling's Physiological Basis of Rehabilitation Medicine. $3^{\text {rd }}$ Ed. Wildwood avenue: Butterworth-Heinemann. pp 397-416

Kim, W.S. and Kim, M.J., 2017. Individual joint contribution to body weight support in the affected lower limb during walking in poststroke hemiplegia. Topics in stroke rehabilitation, 24(3), pp.170-176.

Lloyd A, Bannigan K, Sugavanam T, dan Freeman J, 2018. Experiences of stroke survivors, their families and unpaid carers in goal setting within stroke rehabilitation. JBI Database of Systematic Reviews and Implementation Reports; 16(6), 1418-1453. doi:10.11124/JBISRIR-2017-003499

Li S, Francisco GE, dan Zhou P, 2018. Post-stroke Hemiplegic Gait: New Perspective and Insights. Frontiers in Physiology; 9. doi:10.3389/fphys.2018.01021

Lin SI, 2005. Motor function and joint position sense in relation to gait performance in chronic stroke patients. Archives of Physical Medicine and Rehabilitation; 86(2), 197-203. doi:10.1016/j.apmr.2004.05.009

Pease SW dan Bowyer LB, 2010. Human walking. In Delisa Physical Medicine and Rehabilitation International. 5th Ed. Philadelphia: Lippincott William \&Wilkins. pp121-137

Piriyaprasarth, P., Morris, M. E., Delany, C., Winter, A., \& Finch, S. (2009). Trials needed to assess knee proprioception following stroke. Physiotherapy Research International, 14(1), 6-16.

Sader T, Godefroy O, Hyra M, Diouf M, Doutrellot PL, Duchossoy C, dan Tasseel-Ponche S, 2018. Gait disorders in subacute stroke: Meta-analysis and case series. Annals of Physical and Rehabilitation Medicine; 61, e200. doi:10.1016/j.rehab.2018.05.459

Sullivan JK. 2009. Walking Recovery and Rehabilitation after Stroke. In: Harvey Stroke Recovery and Rehabilitation. Demos Medical. Pp 323-43

Stein J dan Brandstater ME, 2010. Stroke Rehabilitation. In: Delisa's Physical Medicine and Rehabilitation. $5^{\text {th }}$ Ed. Philadelphia: Lippincott Williams \& Wilkins. pp 551-571

Takeda K, Tanabe S, Koyama S, Shomoto K, Naoi Y, Sakurai H, dan Kanada Y, 2018. Relationship between the rate of force development in knee extensor muscles and gait speed in patients with chronic stroke: A cross-sectional study. NeuroRehabilitation; 1-6. doi:10.3233/NRE-182455

Whittle, MW. 2007. Gait Analysis An Introduction. $4^{\text {th }}$ Ed. Philadelphia, Elsevier, Ltd. p223

Zorowitz RD, E Baerga, SJ Cuccurullo, 2015. Stroke. In: Sara J Cuccurullo: Physical Medicine and Rehabilitation Board Review. $3^{\text {rd }}$ Ed. , New York: Demos Medical Publishing LLC. 